\title{
Orientifold Planar Equivalence: The Quenched Meson Spectrum
}

\author{
Biagio Lucini* \\ School of Physical Sciences, Swansea University, Singleton Park, Swansea SA2 8PP, UK \\ E-mail: b. lucinieswansea.ac.uk
}

\section{Gregory Moraitis}

School of Physical Sciences, Swansea University, Singleton Park, Swansea SA2 8PP, UK

E-mail: pygmeswansea.ac.uk

\section{Agostino Patella}

CERN, Physics Department, 1211 Geneva 23, Switzerland

E-mail: agostino.patella@cern.ch

\section{Antonio Rago}

Department of Physics, Bergische Universität Wuppertal, Gaussstr. 20, D-42119 Wuppertal, Germany

E-mail: rago@physik.uni-wuppertal.de

\begin{abstract}
A numerical study of Orientifold Planar Equivalence is performed in SU(N) Yang-Mills theories for $N=2,3,4,6$. Quenched meson masses are extracted in the antisymmetric, symmetric and adjoint representations for the pseudoscalar and vector channels. An extrapolation of the vector mass as a function of the pseudoscalar mass to the large- $N$ limit shows that the numerical results agree within errors for the three theories, as predicted by Orientifold Planar Equivalence. As a byproduct of the extrapolation, the size of the corrections up to $O\left(1 / N^{3}\right)$ are evaluated. A crucial prerequisite for the extrapolation is the determination of an analytical relationship between the corrections in the symmetric and in the antisymmetric representations, order by order in a $1 / N$ expansion.
\end{abstract}

The XXVIII International Symposium on Lattice Field Theory, Lattice2010

June 14-19, 2010

Villasimius, Italy

\footnotetext{
* Speaker.
} 


\section{Introduction}

Orientifold Planar Equivalence [1] is a powerful analytical tool that establishes the equality of certain observables (among which, meson masses) in two classes of gauge theories at $N=\infty, N$ being the number of colours. This equivalence is of particular interest when the two gauge theory families it relates are $\mathrm{SU}(N)$ with $N_{f}$ (anti)symmetric Dirac fermion flavours and $\mathrm{SU}(N)$ with $N_{f}$ Majorana flavours in the adjoint representation. In fact, this special case enables us to transcribe SUSY results to QCD [目], provided that the latter is close (in the sense of the $1 / N$ expansion) to its large- $N$ limit. Some issues related to the equivalence, like e.g. the size of the finite $N$ corrections, mandate an ab-initio calculation. A full dynamical calculation is expensive from a computational point of view. In fact, the cost of the computation is brought almost entirely by the inversion of the fermionic matrix, which for theories with two-index representation fermions requires $O\left(N^{4}\right)$ operations. Before undertaking a full dynamical study, it is convenient to consider the quenched case as a prototype example. Although for theories in two-index representations the quenched theory and the dynamical theory do not coincide in the large- $N$ limit, Orientifold Planar Equivalence still holds in the quenched case, which then becomes a useful toy model to study techniques that could be used in the dynamical investigation. In this spirit, a first paper appeared in which the chiral condensate was shown to coincide in the large- $N$ limit of the adjoint, symmetric and antisymmetric representations [3]. A crucial point in that work was the separation of the corrections in even and odd powers of $1 / N$ by combining the numerical results in the symmetric and antisymmetric representations. As a result, a precise extrapolation to the large- $N$ limit could be performed and Orientifold Planar Equivalence was verified to a high degree of accuracy. Moreover, this technique allowed a computation of corrections up to $O\left(1 / N^{3}\right)$ in the (anti)symmetric representation while still using a reasonably small value of $N$. In this work, we will perform a similar study for the quenched meson spectrum. First, we will show that there is a simple order by order relationship between the coefficients in a large- $N$ expansion of amplitudes in correlation functions and of spectral masses in the symmetric and antisymmetric representations. Then, we shall use a simple chiral ansatz to establish a relationship between the mass of the vector meson $m_{V}$ and the mass of the pseudoscalar meson $m_{P S}$ at finite $N$, for $N$ ranging from two to six, in the adjoint, in the symmetric and antisymmetric representations. Finally, using the analytical relationships between the corrections, the chiral ansatz will be extrapolated to the large- $N$ limit and Orientifold Planar Equivalence proven to hold. The results we will present in this work have already appeared in [ 4 ], to which we refer for a more detailed discussion.

\section{Meson masses for two-index representation fermions in the $1 / N$ expansion}

We extract meson masses from the large distance exponential decay of the meson correlation functions. For the fermion sources we use the Wilson formulation, in terms of which the Dirac operator is given by

$$
D_{x y}=(m+4 r) \delta_{x y}-K_{x y},
$$

with $m$ the bare quark mass and

$$
K_{x y}=-\frac{1}{2}\left[\left(r-\gamma_{\mu}\right) R\left[U_{\mu}(x)\right] \delta_{y, x+\hat{\mu}}+\left(r+\gamma_{\mu}\right) R\left[U_{\mu}^{\dagger}(y)\right] \delta_{y, x-\hat{\mu}}\right] .
$$


In the previous formulae, $x$ and $y$ are site indices and the $\gamma_{\mu}$ the Dirac $\Gamma$ matrices in Euclidean space. $R\left[U_{\mu}(x)\right]$ is the link variable stemming from $x$ in the direction $\hat{\mu}$ expressed in the representation $R$. In our case, $R$ can be the adjoint (Adj), the two-index symmetric (S) or the two-index antisymmetric (AS) representation. For the representation $R$, meson correlators take the form

$$
C_{\Gamma_{1} \Gamma_{2}}^{R}(x, y)=r_{R}\left\langle\operatorname{tr}_{R}\left(D_{y x}^{-1} \Gamma_{1}^{\dagger} D_{x y}^{-1} \Gamma_{2}\right)\right\rangle_{Y M},
$$

where $\operatorname{tr}_{R}$ indicates the trace over the color indices in the representation $R$ and $\Gamma_{1}$ and $\Gamma_{2}$ are combinations of $\Gamma$ matrices associated with the quantum numbers $J^{P C}$ of the mesons. With the choice $r_{R}=1$ for $R=\mathrm{S} / \mathrm{AS}$ and $r_{R}=1 / 2$ for $R=\mathrm{Adj}$, in the large- $N$ limit we have the equality of correlators in these three representations. The factor $r_{R}=1 / 2$ in the adjoint representation can be understood considering that in our formulation we are always taking Dirac flavours in the adjoint, and one Dirac flavour is equivalent to two Majorana flavours.

The formal proof of the equality of the correlators can be sketched as follows. We start expanding the correlators in Wilson loops:

$$
\frac{1}{N^{2}} C_{\Gamma_{1} \Gamma_{2}}^{R}(x, y)=\frac{r_{R}}{N^{2}} \sum_{\mathscr{C} \supset(x, y)} \alpha_{\mathscr{C}}\left\langle\operatorname{tr}_{R} W_{\mathscr{C}}\right\rangle
$$

$\mathscr{C}$ being a closed curve that contains both $x$ and $y$. The coefficient $\alpha_{\mathscr{C}}$ in (2.4) does not depend on the representation. Writing explicitly the traces in two-index representations in terms of the trace of the fundamental representation yields

$$
\begin{aligned}
& \frac{1}{N^{2}} C_{\Gamma_{1} \Gamma_{2}}^{\mathrm{S} / \mathrm{AS}}(x, y)=\frac{1}{2} \sum_{\mathscr{C} \supset(x, y)} \alpha_{\mathscr{C}} \frac{\left\langle\left[\operatorname{tr} W_{\mathscr{C}}\right]^{2}\right\rangle \pm\left\langle\operatorname{tr}\left[W_{\mathscr{C}}^{2}\right]\right\rangle}{N^{2}} \\
& \frac{1}{N^{2}} C_{\Gamma_{1} \Gamma_{2}}^{\mathrm{Adj}}(x, y)=\frac{1}{2} \sum_{\mathscr{C} \supset(x, y)} \alpha_{\mathscr{C}} \frac{\left\langle\left|\operatorname{tr} W_{\mathscr{C}}\right|^{2}\right\rangle-1}{N^{2}}
\end{aligned}
$$

Note that the factors of $1 / 2$ in the two previous formulae have a different origin: for the S/AS, it comes when expressing the trace in the two-index representations in terms of the group element in the fundamental representation, while for the adjoint is the factor $r_{R}$. At large $N$, we can use factorisation and neglect the subleading terms, obtaining

$$
\begin{aligned}
\frac{1}{N^{2}} C_{\Gamma_{1} \Gamma_{2}}^{\mathrm{S} / \mathrm{AS}}(x, y) & =\frac{1}{2} \sum_{\mathscr{C} \supset(x, y)} \alpha_{\mathscr{C}} \frac{\left\langle\operatorname{tr} W_{\mathscr{C}}\right\rangle\left\langle\operatorname{tr} W_{\mathscr{C}}\right\rangle}{N^{2}}, \\
\frac{1}{N^{2}} C_{\Gamma_{1} \Gamma_{2}}^{\mathrm{Adj}}(x, y) & =\frac{1}{2} \sum_{\mathscr{C} \supset(x, y)} \alpha_{\mathscr{C}} \frac{\left\langle\operatorname{tr} W_{\mathscr{C}}\right\rangle\left\langle\operatorname{tr} W_{\mathscr{C}}^{\dagger}\right\rangle}{N^{2}} .
\end{aligned}
$$

Finally, the equality

$$
\lim _{N \rightarrow \infty} \frac{1}{N^{2}} C_{\Gamma_{1} \Gamma_{2}}^{\mathrm{S} / A S}(x, y)=\lim _{N \rightarrow \infty} \frac{1}{N^{2}} C_{\Gamma_{1} \Gamma_{2}}^{\mathrm{Adj}}(x, y)
$$

follows from charge conjugation invariance, which dictates $\left\langle\operatorname{tr} W_{\mathscr{C}}^{\dagger}\right\rangle=\left\langle\operatorname{tr} W_{\mathscr{C}}\right\rangle$. This last step in Yang-Mills theories does not present the conceptual issues discussed in [5, 6] for the dynamical 
case. The equality of the meson spectra in the three theories follows from the equality of the correlators.

Correlators of mesons in the adjoint representation are expected to be expandable in a power series of $1 / N^{2}$. As for the other two representations, since in the symmetric and antisymmetric representations the static sources introduce $1 / N$ effects, there is no reason not to expect $1 / N$ corrections in the meson mass spectrum. Assuming that there is no accidental degeneracy at large- $N$ in the theory with fermions in the antisymmetric and in the symmetric ${ }^{1}$, the masses $m_{R}$ and the amplitudes $A_{R}$ in correlators of fermion bilinears in these two representations can be shown to be related by

$$
A_{\mathrm{S}}^{j}(N)=A_{\mathrm{AS}}^{j}(-N) \quad \text { and } \quad m_{\mathrm{S}}^{j}(N)=m_{\mathrm{AS}}^{j}(-N)
$$

for states $j$ related by Orientifold Planar Equivalence. Order by order in a $1 / N$ expansion, this implies that even-power terms in $1 / N$ in the symmetric and antisymmetric representation have the same coefficient, while odd-power terms have opposite coefficients. This means that the combinations

$$
M^{j}=\left(m_{\mathrm{S}}^{j}+m_{\mathrm{AS}}^{j}\right) / 2 \quad \text { and } \quad \mu^{j}=N\left(m_{\mathrm{S}}^{j}-m_{\mathrm{AS}}^{j}\right) / 2
$$

have an expansion in powers of $1 / N^{2}$.

\section{Numerical results}

We generated gauge configurations for values of $N$ ranging from two to six using the standard Wilson action. Both the generation of the configurations and the analysis of the correlators in two-index representations were performed using the purposely developed HiRep code [7]. The technical details of the simulations have been given in [ $₫$. Here we remark that we simulate at the same $\beta$ values as in Ref. [3], where the couplings were chosen so that the gauge theories at various $N$ are at a common value of the lattice spacing $a=1 /\left(5 T_{c}\right), T_{c}$ being the deconfinement temperature of the pure gauge system [8].

After extracting $m_{V}$ and $m_{P S}$, we have determined the chiral limit value of $m_{V}$ (denoted as $m_{V}^{\chi}$ ) using the chiral ansatz

$$
m_{V}\left(m_{P S}\right)=m_{V}^{\chi}+B m_{P S}^{2} .
$$

Our results and the corresponding fits are displayed in Figs. 1, 2 and 3 respectively for the adjoint, symmetric and antisymmetric representations. We note that there is a well-defined hierarchy for $m_{V}$ at fixed $m_{P S}$, with the symmetric mass being the highest and the antisymmetric being the lowest. At fixed representation, the vector mass in the antisymmetric has the largest variation with $N$, while there is little or no variation with $N$ for the adjoint vector masses.

The large- $N$ extrapolation has been performed for the quantity $m_{V, A d j}^{\chi}$ and for the quantities $M_{V}^{\chi}$ and $\mu_{V}^{\chi}$ (see Eqs. (2.11) ) using in all cases only the leading term and the $1 / N^{2}$ correction. The

\footnotetext{
${ }^{1}$ In Quantum Field Theory, degeneracies are due to a symmetry, and there is no symmetry that could generate degeneracies in these theories.
} 


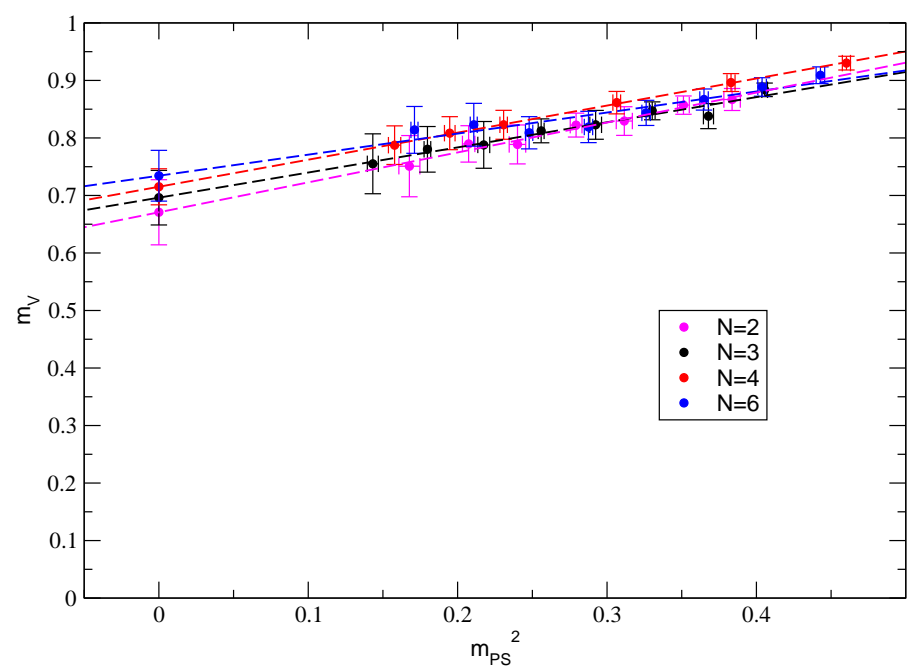

Figure 1: $m_{V}$ as a function of $m_{P S}^{2}$ for the adjoint representation at the values of $N$ shown. The solid lines are chiral fits to the data.

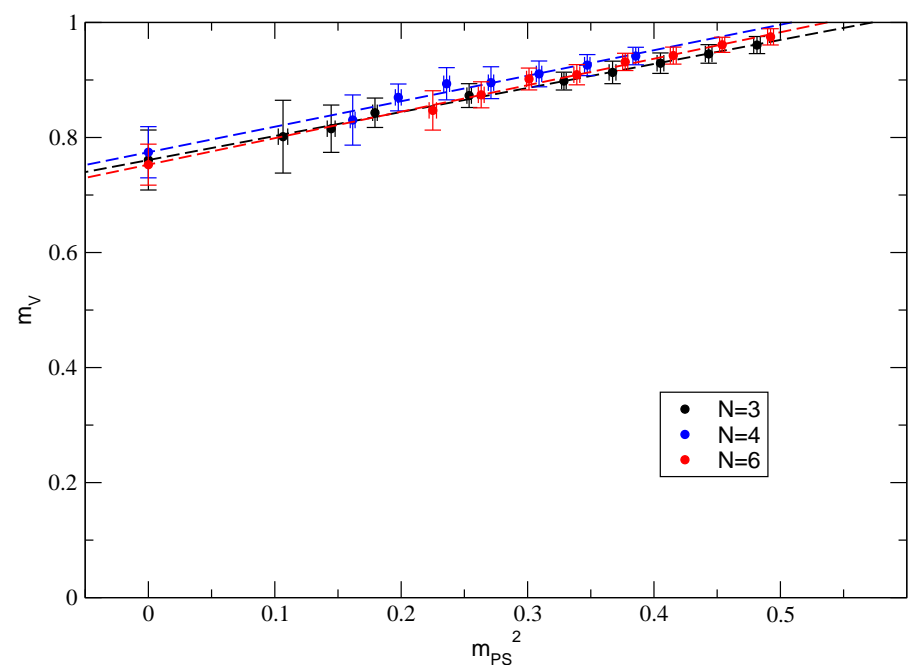

Figure 2: $m_{V}$ as a function of $m_{P S}^{2}$ for the symmetric representation at the values of $N$ shown. The solid lines are chiral fits to the data.

quality of the fits is displayed in Fig. $甘$. The values of $m_{V, \mathrm{~S}}^{\chi}$ and $m_{V, \mathrm{AS}}^{\chi}$ as a function of $1 / N$ are obtained inverting Eqs. (2.11). Our results are:

$$
\begin{gathered}
m_{V, \mathrm{Adj}}^{\chi}=0.736(17)-\frac{0.28(11)}{N^{2}}, \\
m_{V, \mathrm{~S}}^{\chi}=0.723(27)+\frac{0.40(11)}{N}-\frac{1.02(32)}{N^{2}}+\frac{0.3(1.2)}{N^{3}}, \\
m_{V, \mathrm{AS}}^{\chi}=0.723(27)-\frac{0.40(11)}{N}-\frac{1.02(32)}{N^{2}}-\frac{0.3(1.2)}{N^{3}} .
\end{gathered}
$$

The equality of the large- $N$ results for the three representations is a prediction of Orientifold Planar 


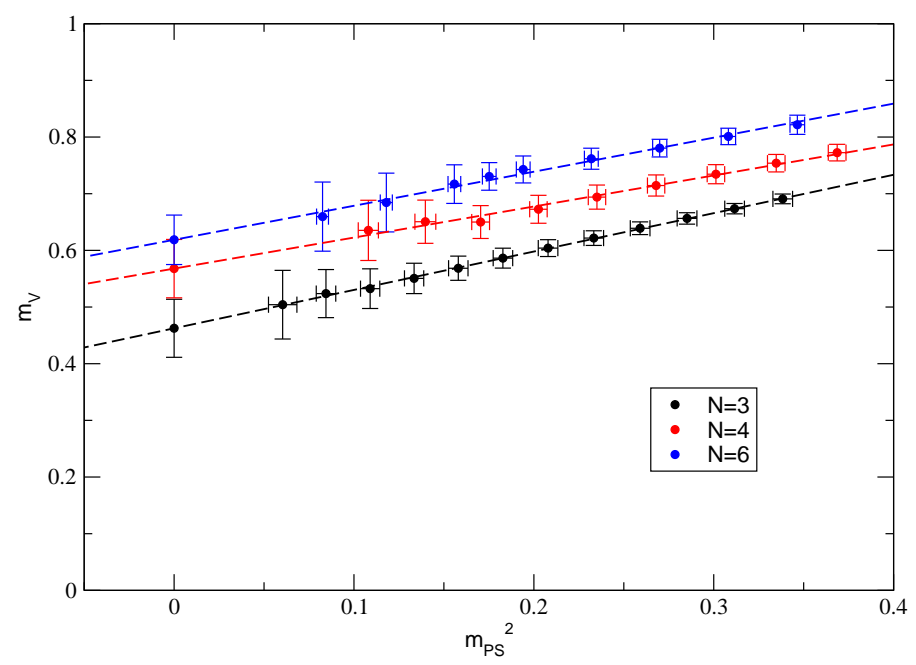

Figure 3: $m_{V}$ as a function of $m_{P S}^{2}$ for the antisymmetric representation at the values of $N$ shown. The solid lines are chiral fits to the data.

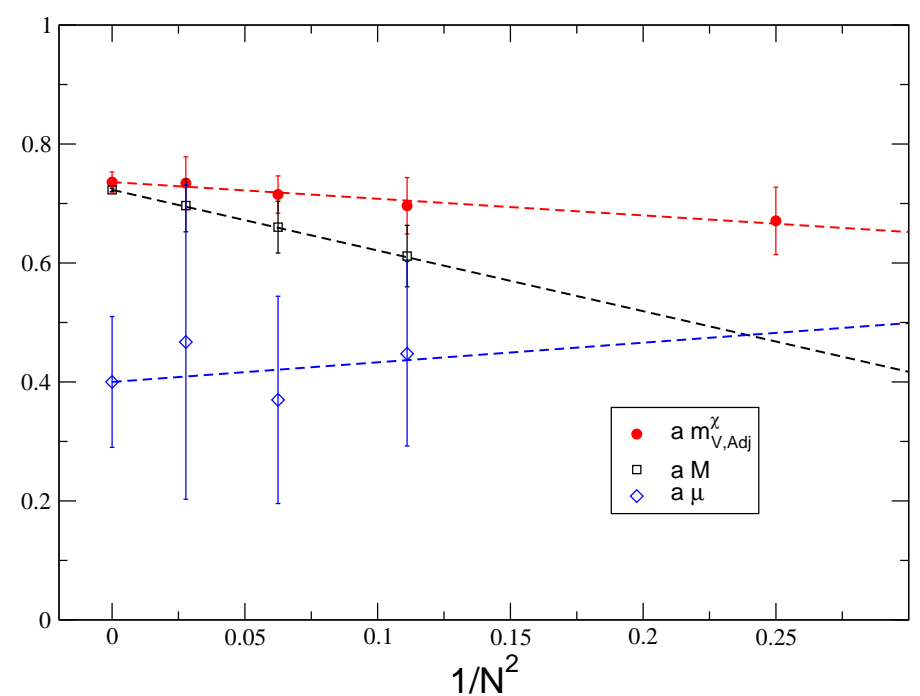

Figure 4: Large- $N$ extrapolation of the adjoint mass and of the combinations $M$ and $\mu$, all in the chiral limit.

Equivalence. We remark that through the separations of corrections in even and odd powers of $1 / N$ in the symmetric and antisymmetric representation, we were able to get an handle on the size of the corrections up to $O\left(1 / N^{3}\right)$. Numerically, Orientifold Planar Equivalence can be verified for any value of $m_{P S}$ in the chiral region [4].

\section{Conclusions}

Our work should be regarded as an illustration of a possible strategy for a numerical proof of Orientifold Planar Equivalence. We have shown that it is possible to combine the data in the symmetric and antisymmetric representation for extracting precise values in the large- $N$ limit from 
data at moderately small values of $N$. The next step in this investigation is the simulation of the dynamical theories.

\section{Acknowledgments}

We thank Luigi Del Debbio and Claudio Pica for the collaboration that lead to the development of the HiRep Monte Carlo code used in this work. Numerical simulations have been performed on a 120 core Beowulf cluster partially funded by the Royal Society and STFC and on the IBM Blue/C system at Swansea University. The work of B.L. is supported by the Royal Society through the University Research Fellowship scheme and by STFC under contract ST/G000506/1. A.R. thanks the Deutsche Forschungsgemeinschaft for financial support. A.P. was supported by the European Community - Research Infrastructure Action under the FP7 "Capacities" Specific Programme, project "HadronPhysics2".

\section{References}

[1] A. Armoni, M. Shifman and G. Veneziano, Exact results in non-supersymmetric large N orientifold field theories, Nucl. Phys. B 667, 170 (2003) [arXiv: hep-th/0302163]; Refining the proof of planar equivalence, Phys. Rev. D 71, 045015 (2005) [arXiv: hep-th/ 0412203 ].

[2] A. Armoni, M. Shifman and G. Veneziano, SUSY relics in one-flavor QCD from a new 1/N expansion, Phys. Rev. Lett. 91, 191601 (2003) [arXiv: hep-th/0307097];

[3] A. Armoni, B. Lucini, A. Patella and C. Pica, Lattice Study of Planar Equivalence: The Quark Condensate, Phys. Rev. D 78 (2008) 045019 [arXiv:0804.4501 [hep-th]].

[4] B. Lucini, G. Moraitis, A. Patella and A. Rago, A numerical investigation of orientifold planar equivalence for quenched mesons, arXiv:1008.5180 [hep-lat].

[5] M. Unsal and L. G. Yaffe, (In)validity of large N orientifold equivalence, Phys. Rev. D 74, 105019 (2006) [arXiv:hep-th/0608180].

[6] A. Armoni, M. Shifman and G. Veneziano, A note on C-Parity Conservation and the Validity of Orientifold Planar Equivalence, Phys. Lett. B 647, 515 (2007) [arXiv : hep-th/0701229].

[7] L. Del Debbio, A. Patella and C. Pica, Higher representations on the lattice: numerical simulations. SU(2) with adjoint fermions, Phys. Rev. D 81, 094503 (2010) [arXiv:0805.2058 [hep-lat]].

[8] B. Lucini, M. Teper and U. Wenger, The high temperature phase transition in $S U(N)$ gauge theories, JHEP 0401, 061 (2004) [arXiv: hep-lat/ 0307017 ]. 